\title{
Explanation of Molecular Processes without Tracking Mechanism Operation
}

\section{Ingo Brigandt}

To contact the author, please write to: Department of Philosophy, University of Alberta, 2-40 Assiniboia Hall, Edmonton, AB T6G2E7, Canada; email: brigandt@ualberta.ca.

\begin{abstract}
Philosophical discussions of systems biology have enriched the notion of mechanistic explanation by pointing to the role of mathematical modeling. However, such accounts still focus on explanation in terms of tracking a mechanism's operation across time (by means of mental or computational simulation). My contention is that there are explanations of molecular systems where the explanatory understanding does not consist in tracking a mechanism's operation and productive continuity. I make this case by a discussion of bifurcation analysis in dynamical systems, articulating the distinctive way in which explanatory understanding is provided, especially about the reversibility or irreversibility of molecular processes.
\end{abstract}

Forthcoming in Philosophy of Science 


\section{Acknowledgements}

Earlier versions of this paper have been presented to audiences at the S-ANU meeting 2015, ISHPSSB 2015, CSHPS 2016, and PSA 2016. Special thanks go to Arnon Levy and Bill Bechtel for submitting the PSA 2016 symposium proposal. I am also grateful to Arnon Levy and two reviewers for comments on a written version of this paper. The work on this essay was supported by the Social Sciences and Humanities Research Council of Canada (Insight Grant 435-20160500). 


\section{Introduction}

Mechanisms and mechanistic explanation continue to be core issues for contemporary philosophy of biology. Beyond the focus on mechanistic research in the style of classical molecular biology (Craver and Darden 2013), many recent philosophical discussions have begun to call attention to scientific studies of large-scale networks and complex molecular processes in the domain of systems biology, which often involve computational and mathematical modeling tools. Some philosophers have concluded that the theories of systems biology do not qualify as mechanistic (Braillard 2010; Issad and Malaterre 2015). Others have granted that systems biology is in continuity with paradigmatic mechanistic research, while likewise underscoring that this biological domain contains distinctive types of theorizing that simply have not been addressed by prior philosophical accounts of mechanisms (Brigandt et al. 2017; Green et al. 2017; Levy and Bechtel 2016). My account will be in the latter spirit, as I deem it to be more insightful to articulate diverse ways of biological theorizing (especially those that have been neglected by philosophers) than to defend a uniquely correct notion of 'mechanistic.' Indeed, quite different things can be addressed under the mechanism label: whether scientists' particular objects of investigation are mechanisms, whether an explanation put forward is mechanistic, or whether the experimental methods or reasoning strategies employed are instances of mechanistic research.

The topic of my discussion is explanation. Recent philosophical discussions of systems biology have from the outset emphasized the important role of mathematical modeling as something neglected by previous accounts of mechanistic explanation (e.g., Bechtel and Abrahamsen 2010; Brigandt 2013). Yet my contention is that even these accounts have focused on what is only one type of model-based explanation. Their (perfectly legitimate) starting point 
has been that in addition to pointing to the components and organization of a mechanism, a mechanistic explanation also has to epistemically lay out how the operation of the mechanism generates the phenomenon to be explained. In the case of a relatively simple mechanism, this explanatory understanding comes from mentally simulating the mechanism's operation, where this mental simulation can be aided by a mechanism diagram (Bechtel 2011; Bechtel and Abrahamsen 2005; Brigandt 2013; Levy and Bechtel 2013). ${ }^{1}$ In the case of a mechanism whose behavior depends on quantitative interactions or its complex organization (e.g., feedback loops), the explanation laying out how the explanandum phenomenon is generated is provided instead by a computer simulation: "the purpose of a computational simulation (like mental simulation in the basic mechanistic account) is ... to determine whether the proposed mechanism would exhibit the phenomenon" (Bechtel 2011, 553). Bechtel and Abrahamsen (2010) have dubbed this type of explanation 'dynamic mechanistic explanation,' and I myself have echoed this vision: "the explanation has to show how (or at least that) the explanandum results from this. If the mechanism's operation cannot be understood by mental simulation, ... a computer simulation is needed" (Brigandt 2015, 161).

Although such recent accounts have pointed to one important way in which mathematical modeling in systems biology generates explanations, by effectively equating the providing of explanatory understanding with tracking a mechanism's operation (up to the explanandum phenomenon resulting), these accounts have thereby focused on a computer simulation

1 "When mechanisms are organized in this [simple] way, basic mechanistic explanation, in which one characterizes the functioning of the overall mechanism qualitatively in terms of the contribution of each of its parts and mentally rehearses (simulates) how it produces the phenomenon, suffices." (Bechtel 2012, 235) 
representing how a system's components and variables change across time. ${ }^{2}$ In contrast, I argue that there are various ways in which the analysis of mathematical models in systems biology provides explanatory understanding without tracking a mechanism's operation and productive continuity. Something analogous has been previously maintained by Huneman (2010), in the context of what he calls topological explanations, which explain in terms of a network's abstract structure. Although Levy and Bechtel (2013) deem explanations in terms of a system's structural organization to be mechanistic, Huneman claims topological explanations to be non-mechanistic on the grounds that they neither list specific activities nor appeal to a temporal sequence of events. The latter is similar to my perspective, though rather than pointing to examples of 'explanation that is non-mechanistic' I more precisely argue for 'explanation without tracking mechanism operation.' More importantly, whereas Huneman addresses explanation in terms of a network's static structure, my discussion crucially involves the explanation of system dynamics.

In what follows, I will discuss the mathematical analysis of dynamical models. Although there are in fact different kinds of model analysis, resulting in different types of explanation, in this paper I can only focus on one type, namely, bifurcation analysis. I will explain what kind of important explanatory understanding is provided by bifurcation analysis without tracking a mechanism's operation across time (as would be done by a computer simulation).

\section{Different Types of Bifurcations}

Like many other models in systems biology, dynamical models are developed based on

\footnotetext{
2 "computational simulations ... involves writing differential equations describing how the rates of individual operations are affected by various parameters and using computers to show how a system involving such operations would change over time" (Bechtel 2010, 320-21).
} 
mechanistic information of the system at hand, such as mechanism diagrams or more complex reaction networks showing which gene products, enzymes, or metabolites interact with each other. The variables of the model are often the concentrations of such entities within a cell. Ordinary or partial differential equations are used to capture how due to molecular interactions a change in one variable results from changes in other variables, where the reaction kinetics parameters are experimentally known or are estimated by fitting them to limited experimental data. This at least models a system's change across time, although occasionally spatial changes (e.g., across different cells) are captured as well.

In addition to mathematically analyzing the properties of dynamical systems, one way of visually representing some relevant features is in terms of phase spaces. A phase space is a multidimensional space where each axis represents one system variable, such as the concentration of an enzyme or metabolite within a cell. The phase space encompasses all possible system states (each point of the space is one possible state), so that a system's dynamic change across time is a point moving along a trajectory within phase space. A few of these dimensions and thus selected aspects of a system can be visually represented. Figures 1 and 2 are phase spaces, where the 'Input' and 'Output' axes represent two system variables, while in Figure 3 all axes represent system variables. Note that such phase spaces are not mechanism diagrams. Phase spaces do not represent mechanism entities (e.g., a certain molecule), but possible system states (the possible concentrations of a molecule), and they do not capture the spatial organization and concomitant interactions of mechanism entities.

A steady state is any state of a system which when reached will not change. There are stable steady states, to which the system will converge from anywhere in the steady state's vicinity, so that the system will return to this state even when perturbed. This is often visually captured by a 
diagram in which the vertical axis represents the system's energy state $\Phi$, as in Figures 1 and 2, where the dynamic behavior of the system is 'downwards' towards lower energy states and the stable steady states show up as the bottom of a valley. There are also unstable steady states, which show up as the top of a hill. If the system is exactly in such a state, it will remain there, but the slightest perturbation (e.g., due to molecular noise inside a cell) will make the system move away from this unstable steady state. Some systems have just one stable steady state and are called monostable. Systems that have two stable steady states (valleys) separated by a hill (an unstable steady state) are called bistable (Ferrell 2012). Bistability is of biological significance as even though each of the two stable cellular states is maintained in the face of cellular noise, transitions between these states are possible (if other system components move it across the hill).

A bifurcation is a location in phase space exhibiting a qualitative change in topology and thus dynamics of the system, such as a change in the number and types of steady states. Figure 1 depicts a pitchfork bifurcation, in which a monostable configuration (upper part) changes at $\Psi$ to a bistable configuration. The black solid lines represent stable steady states, while the dashed line depicts an unstable steady state (a hilltop in between two stable steady states). This kind of topology is well-known from Waddington's epigenetic landscapes, which depict development as a series of branching events leading to different possible developmental outcomes. But note that a pitchfork bifurcation yields a reversible process: the system starts out in one steady state, but regardless of whether the left or right arm of the bifurcation was chosen after an increase in the input (the concentration of a cellular entity controlling the system), when the input is reduced again the system ends up in the very steady state where it started out. Such a reversible bifurcation would not be ideal and may not actually obtain for many of the instances of developmental differentiation that Waddington had in mind (but see Ferrell 2012 for examples exhibiting this type of bifurcation). 


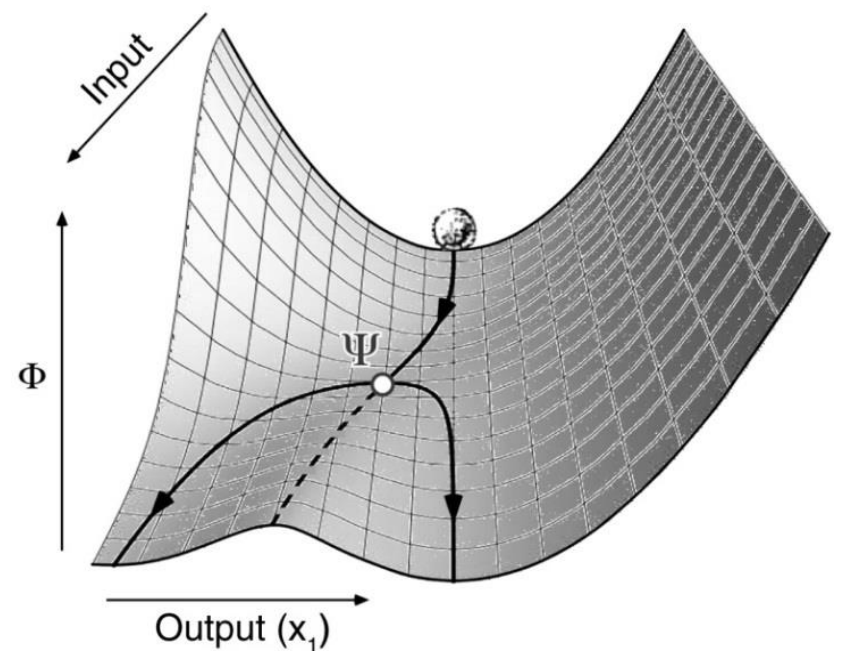

Figure 1: A pitchfork bifurcation (at $\Psi$ ), yielding a process that is reversible: it returns to the original steady state when input is withdrawn again. Reprinted from Ferrell (2012) with permission from Elsevier.

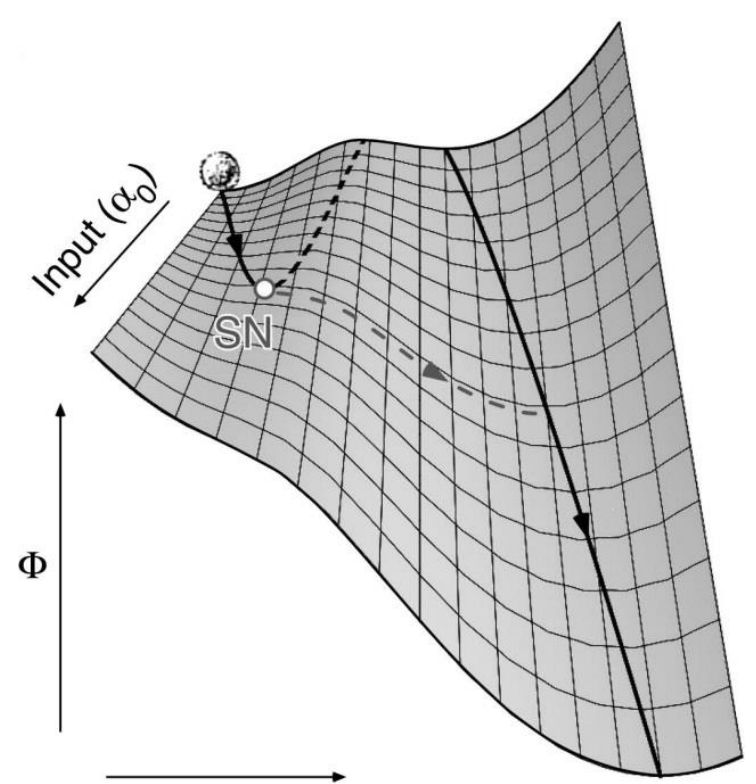

Output (x)

Figure 2: A saddle-node bifurcation (at $\mathrm{SN}$ ), yielding a locally irreversible process: new, high-output steady state is maintained even when input is withdrawn again. From Ferrell (2012) with permission from Elsevier.

The type of bifurcation shown in Figure 2 is theoretically and biologically more interesting. Although it is likewise a transition between a monostable and a bistable configuration, this saddle-node bifurcation is locally irreversible. The black solid and dashed lines represent again the steady states (the unstable ones being at the 'saddle' of the landscape), while the grey dashed line with the arrowhead simply depicts a trajectory of the system. At low concentrations of the input stimulus, the system is bistable, with a steady state on the left (corresponding to low output) and one on the right. Assume that the system starts in the left steady state. As soon as the input is increased past the saddle-node bifurcation point $\mathrm{SN}$, this steady state disappears and the system converges to the other, right-hand side steady state (corresponding to high output). The 
crucial feature - and difference to the above pitchfork bifurcation — is that even if the input stimulus is reduced to the starting condition, the system will remain in the right-hand steady state. The transition from the low-output to the high-output steady state is thus irreversible - it is maintained even if the stimulus which triggered the transition is fully withdrawn (Ferrell 2012).

\section{Biological Examples of Locally or Globally Irreversible Dynamical Profiles}

A bifurcation analysis consists in mathematically investigating a dynamical model to reveal such aspects of its dynamical profile as the number and types of bifurcations, or regions where the system exhibits bistability. (I am calling it a 'dynamical' profile, as it includes knowledge about the direction in which change from a possible state would occur, given the underlying system dynamics.) There are many more types of bifurcations than the two mentioned in the previous section, but now it is time to illustrate bifurcation analysis in a concrete biological case- the progression of the cell cycle. The standard eukaryotic cell cycle consists of the S phase (in which DNA synthesis and replication occurs), followed by the $\mathrm{G}_{2}$ gap phase and the $\mathrm{M}$ phase (mitosis as the division of the cell). Then both daughter cells enter the $\mathrm{G}_{1}$ gap phase (during which the cell size increases), after which we are back to the $\mathrm{S}$ phase. Crucial in the mechanism underlying the progression of the cell cycle are various cyclins and cyclin-dependent kinases, which are synthesized and degraded at different phases.

The example I use is the model of the budding yeast cell cycle by Chen et al. (2000). As in many studies, they investigate various aspects of their model, but my focus will be on the result of the analysis depicted in Figure 3 (for additional background and a similar bifurcation analysis see also Novak et al. 1998). As in the above examples, this phase space depicts stable steady 
states by solid lines and unstable ones by a dashed line. The vertical axis is the output, where a low activity of $\mathrm{Clb}$-dependent kinases corresponds to the $\mathrm{G}_{1}$ phase and a high activity yields the S phase and the following $M$ phase - in yeast there is no recognizable $G_{2}$ phase in between. The horizontal axis depicts the input, using the ratio of two cyclins which trigger the transitions between the phases. In addition to their mathematical analysis, the arrows in the center of the figure also depict how Chen et al. (2000) experimentally confirmed the bistability of the yeast system modeled, where experimental expression of Clb5 and Sic1 results in a shift to the new phase, depending on whether this stimulus is large enough to move the system beyond the unstable steady state.

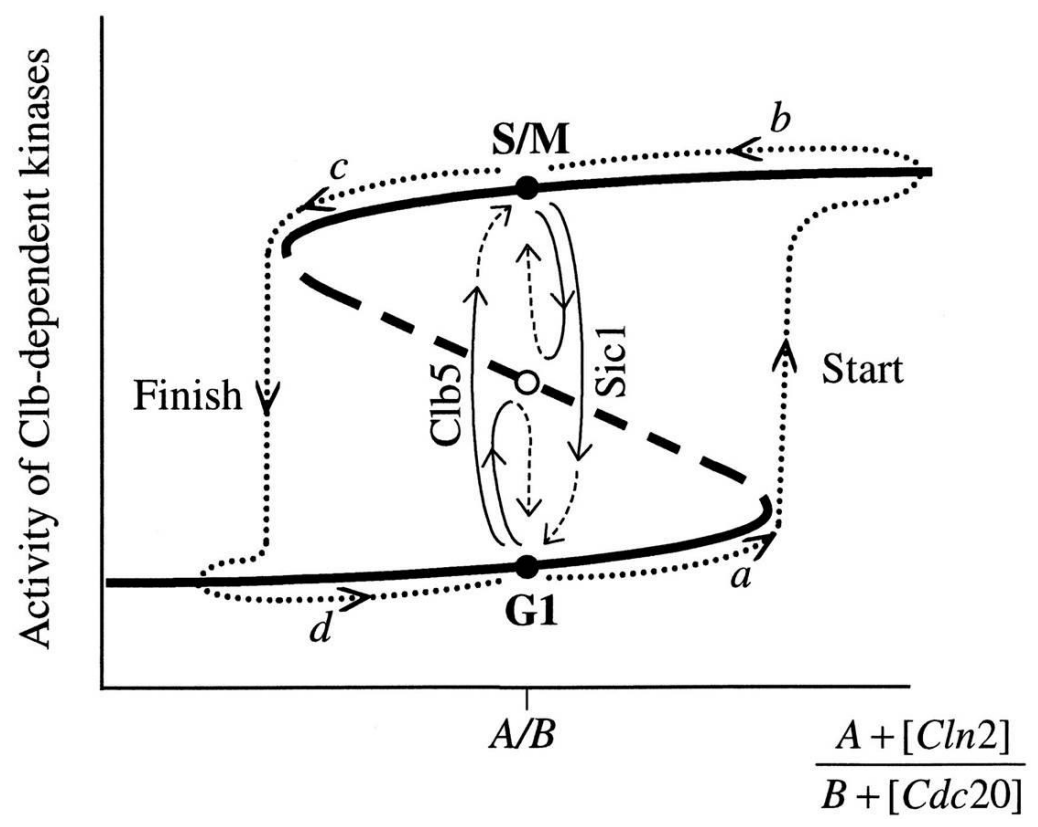

Figure 3: The result of a bifurcation analysis of Chen et al.'s yeast cell cycle model. Cell cycle progression is controlled by the input on the horizontal axis, resulting in the cell being in either the $\mathrm{G}_{1}$ phase or in the $\mathrm{S}$ and following $\mathrm{M}$ phase. The system has two saddle-node bifurcations (where either solid line meets the dashed line), which ensure that a switch to a new cell cycle phase is maintained after removal of the trigger-although this switch can eventually be reversed. Reprinted from Chen et al. (2000) with permission of the American Society for Cell Biology. 
The important feature is the presence of two bifurcations, located where either of the two solid lines meets the dashed line in Figure 3. They are saddle-node bifurcations (the type we encountered in Fig. 2) and thus locally irreversible, which in the present case has the advantage that a switch to the next cell cycle phase is maintained even if there are fluctuations in the factor that triggered the switch (so that there is no constant flip-flopping between phases). Assume the cell is in the $\mathrm{G}_{1}$ phase. A rise in the concentration of cyclin $\mathrm{Cln} 2$ (an increasing value on the horizontal axis) activates a corresponding cyclin-dependent kinase and pushes the state of the system rightward past the point labeled $a$ and one of the bifurcation points, so that the system moves past 'Start' and converges toward a new steady state with a quite different, high concentration of $\mathrm{Clb}$-dependent kinases, thereby entering the $\mathrm{S}$ phase. Underlying cellular processes remove $\mathrm{Cln} 2$ again, but due to the locally irreversible bifurcation the cell remains in this new steady state. To be sure, the overall dynamical profile yields a globally reversible process. Once the cell has made it into the $\mathrm{M}$ phase, a rise in the $\mathrm{Cdc} 20$ concentration (a sufficiently decreasing value on the horizontal axis) pushes the state of the system past point $c$ and the second bifurcation point, so that it converges back to the original steady state corresponding to the $\mathrm{G}_{1}$ phase.

Thus, the locally irreversible bifurcations ensure that the cell progresses to the next phase of the cell cycle (without immediately moving one phase backwards), while the globally reversible configuration is needed to make a cycle possible. ${ }^{3}$ Tracing one system trajectory from $a$ via $b$ and $c$ to $d$ and again to $a$ is indeed an instance of tracking a mechanism's operation, however, the

\footnotetext{
${ }^{3}$ This overall situation is also an instance of what in engineering is known as hysteresis, which is the possibility of moving back and forth between two outcomes, but without unwanted frequent switching.
} 
explanatory question of interest to my discussion is why the system exhibits local irreversibility, yet global reversibility. This involves considerations about what states and changes are possible or impossible for the system, to be explained by the nature of the bifurcations (capturing local dynamical potentialities) and by the global dynamical profile (capturing possible trajectories).

It is instructive to compare this locally irreversible, yet globally reversible dynamical profile with a globally irreversible bifurcation configuration. A case in point is apoptosis, i.e., programmed cell death. Apoptosis of a cell can be triggered by a stimulus from outside of the cell (via the cell's death receptor and the so-called extrinsic pathway), or by a signal at the cell's mitochondria (in response to cytotoxic stress via the intrinsic pathway). Downstream of these triggers, the initiation of apoptosis is molecularly regulated by a network of interactions involving proteases called caspases, among other entities. Focusing on the intrinsic pathway, Legewie et al. (2006) mathematically investigate the role of the X-linked inhibitor of apoptosis protein (XIAP) in regulating apoptosis. The mechanism diagram in the upper right corner of Figure 4 shows their 'wild-type model,' which is most similar to the situation found in nature. The pathway starting at the mitochondria activates Apaf-1, subsequently stimulating the 'initiator' caspase Casp9, which, if leading to a high concentration of the 'effector' caspase active Casp3, results in execution of the downstream apoptosis mechanism. To theoretically scrutinize the role of XIAP, the researchers also modeled modified, counterfactual mechanisms ('mutant models'), one of which is shown in lower right corner of Figure 4-compared to the real mechanism it lacks the Casp9 $\leftarrow$ Casp 3 feedback. $^{4}$

\footnotetext{
${ }^{4}$ While in this as in the wild-type model each XIAP molecule (out of a limited concentration) can inhibit either a Casp9 or a Casp3 molecule, in a second mutant model a XIAP molecule is permitted to
} 


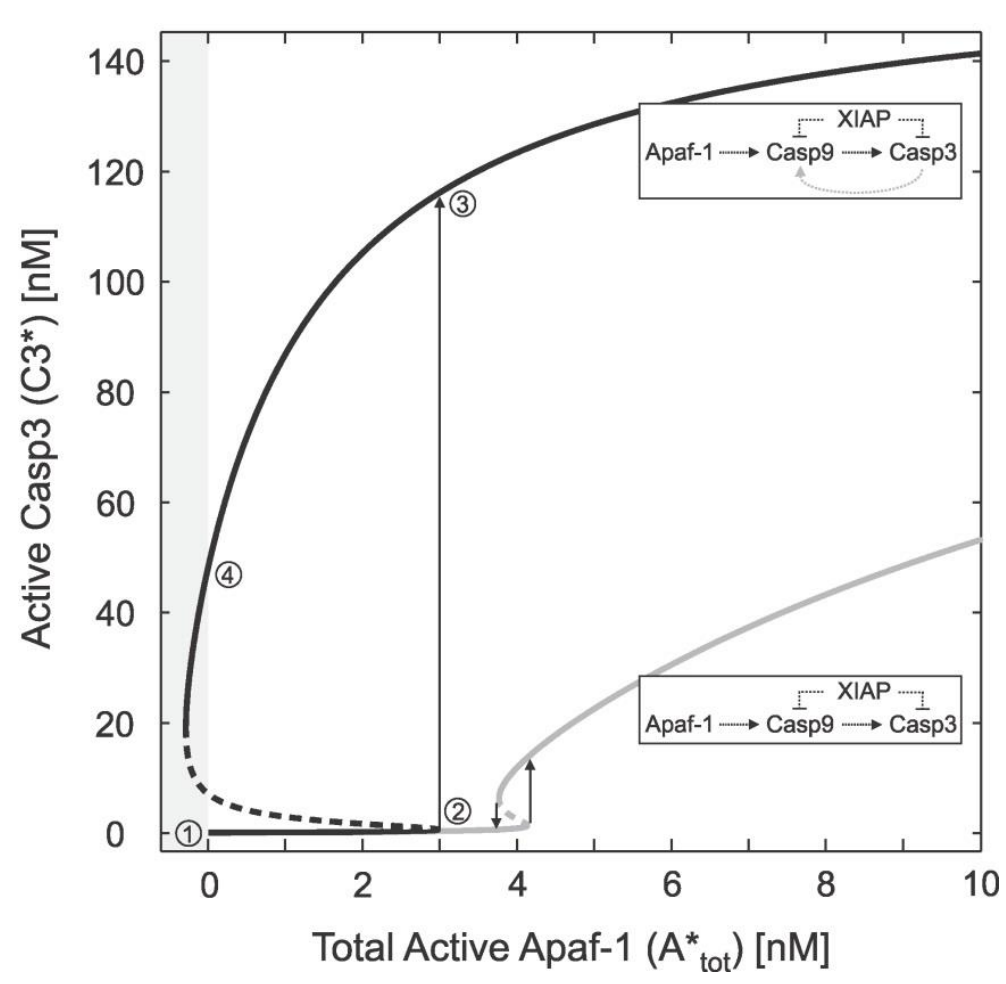

Figure 4: Bifurcation analysis results of Legewie et al.'s apoptosis modeling. The realistic apoptosis regulation mechanism is shown at the top, and it yields the steady state profile depicted in black. A counterfactual mechanism (shown at the bottom) was also modeled, resulting in the profile in grey. While the latter is a globally reversible configuration, bifurcation analysis of the realistic mechanism shows why the transition from the negligible to the high $\mathrm{C} 3 *$ output steady state is globally irreversible. Reprinted from Legewie et al. (2006) with Creative Commons license.

The mutant model yields the globally reversible dynamical profile that Figure 4 depicts in grey. It is exactly the one we have previously encountered in the case of the cell-cycle

bind to Casp9 and Casp3 simultaneously. See Legewie et al. (2006), in particular their Figure 4, for more detailed results (including an explanation of why even the first mutant model without any positive feedback loop yields bistability). Although it can be counted as an instance of mechanistic reasoning, this comparison across theoretically possible systems is not a case of tracking a mechanism's operation across time either. 
progression (Fig. 3) - a biological context where global reversibility is in fact needed. However, a cell's commitment to apoptosis better be irreversible, so that the apoptotic cell's damaged components are fully removed. Irreversibility does in fact obtain for the wild-type model - the actual mechanism. Moreover, the bifurcation analysis explains this global irreversibility by yielding the dynamical profile depicted in black in Figure 4. The model equations also have negative solutions, but negative concentrations (the area with the grey background in Fig. 4) have of course no relevance for the possible behaviors of the biological system. Starting in the steady state with a negligible active Casp3 output, once the active Apaf-1 input increases so as to push the system past the saddle-node bifurcation (the point labelled 2), the system settles in a new steady state with a high active Casp3 output (point 3). Unlike in the earlier situation (Fig. 3), there is no second bifurcation (in the range of positive concentrations), so that the system remains in the new steady state even if the Apaf- 1 input concentration is fully reduced to zero (point 4).

\section{Bifurcation Analysis Explains without Tracking Mechanism Operation}

The motivation for my discussion of bifurcation analyses of dynamical models has been previous accounts along the lines of dynamic mechanistic explanation. Based on the point that in the case of simple mechanisms the explanatory understanding comes from mentally simulating a mechanism's operation (that generates the explanandum phenomenon), these accounts have focused on the analogous situation for complex mechanisms: explaining by tracking a mechanism's operation, as done by a computer simulation of a mathematical model. 


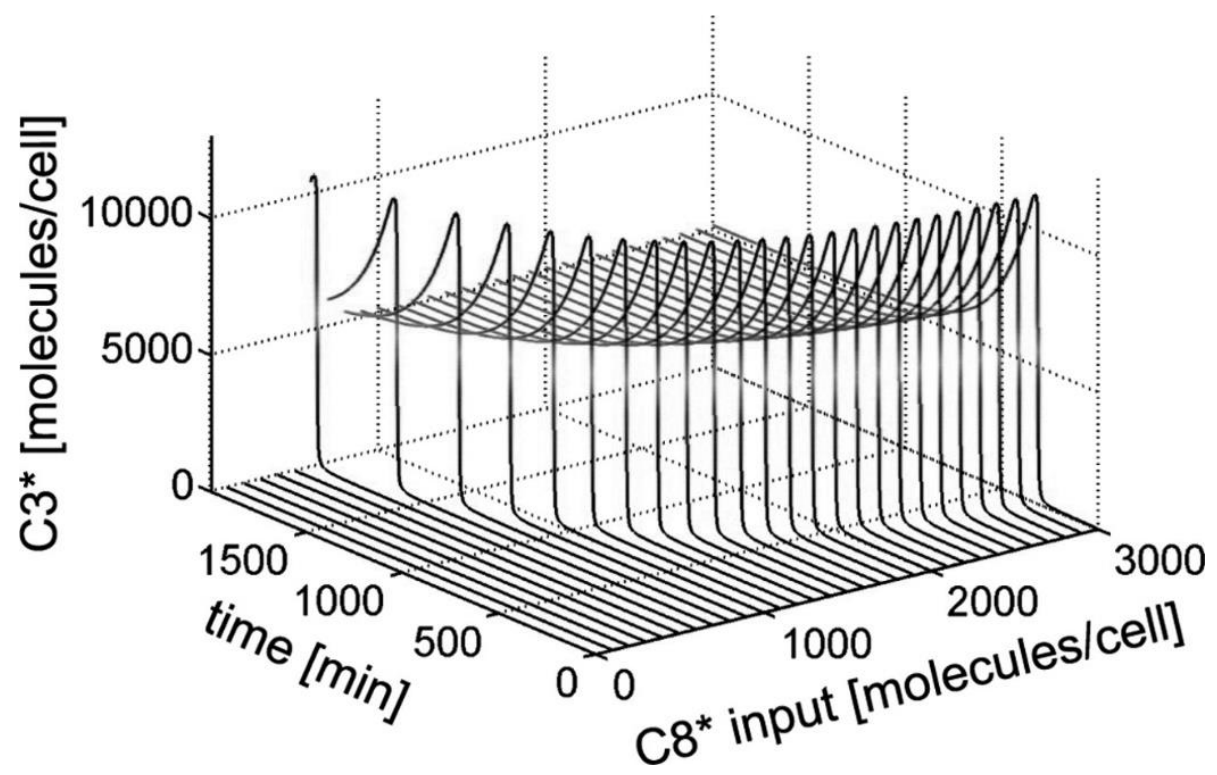

Figure 5: Multiple computer simulations (each starting with a different input concentration) of Eissing et al.'s apoptosis model. A stimulus from the cell's death receptor activates C8* (active Casp8). Reprinted from Eissing et al. (2004) with permission from the American Society for Biochemistry and Molecular Biology.

My central claim is that there are kinds of analyses of mathematical models that also are genuine explanations, yet the explanatory understanding does not consist in tracking a mechanism's operation and productive continuity across time. This argument can be illustrated by comparing computer simulation with bifurcation analysis in the case of apoptosis. Figure 5 depicts the results of several computer simulations (in this case of the extrinsic apoptosis pathway, modeled by Eissing et al. 2004), each simulation starting with a different active Casp8 $\left(\mathrm{C}^{*}\right)$ input concentration. These results suggest bistability (negligible as opposed to high active Casp3 output as the two states); and we learn that once a certain input threshold is crossed, the output will rise and happen to remain at a high concentration. However, even multiple computer simulations do not explain why this is the case. Most importantly, no explanation of 
irreversibility — why a withdrawal of the input would not result in a return to the negligible output state - has been given. In contrast, the bifurcation analysis yielding the dynamical profile shown in black in Figure 4 explains global irreversibility in the manner laid out in the previous section: the bifurcation is locally irreversible and there is no further bifurcation (in the range of non-negative concentrations) that would permit a global reversal. The same holds for explaining why global reversibility holds in other systems (see Figure 3), which is also not about tracking a mechanism's actual operation across time (corresponding to one trajectory in phase space), but consists in an analysis of what system changes are possible or impossible. Furthermore, an explanation of local irreversibility in terms of a saddle-node bifurcation makes plain that more than what is strictly depicted in Figure 3 is involved (Figure 2 alone depicts some additional relevant information), and that the complete explanation includes additional analytical knowledge, for instance about the presence and the nature of saddle-node bifurcations. Generally, bistability, irreversibility, and reversibility have a modal aspect, and their explanation involves local modal features (e.g., the direction in which a system would change from a possible state, or the type of a bifurcation), and sometimes features of the global profile (e.g., the number of bifurcations, and the overall topology of bifurcations and stable and unstable steady states). ${ }^{5}$

When arguing that bifurcation analysis explains in this manner, I do not maintain that there are no other explanations - possibly standard mechanistic ones—being put forward in the scientific studies discussed. For there are even cases where one and the same phenomenon can be

\footnotetext{
${ }^{5}$ Bifurcation analysis may also be an instance of 'structural explanation', which on Huneman's (2017) recent account is an explanation where the explanandum directly follows from a topological or other mathematical property (so that a general mathematical dependency rather than the concrete mechanistic model accounts for the explanandum at hand).
} 
explained in very different, yet legitimate ways, as made plain by Sterelny's (1996) use of the distinction between actual-sequence and robust-process explanations in the context of evolutionary biology. An actual-sequence explanation traces the sequence of phylogenetic modifications up to the explanandum trait—which is analogous to tracking a mechanism's operation. A robust-process explanation, in contrast, accounts for the explanandum trait as having been favored by natural selection, so as to indicate that the outcome would have resulted regardless of deviations from the actual phylogenetic sequence. The above systems biological explanations invoking local or global irreversibility are closer to robust-process explanations. Overall, a mathematical model of a molecular system can be investigated in various ways, resulting in several explanations, and possibly different types of explanations. All these explanations are indeed based on the mechanism's components and its organization (possibly also considering modifications of the mechanism), but what matters for my purposes is how the analysis of some system aspects proceeds, resulting in a distinct way in which explanatory understanding is provided. In the case of bifurcation analysis, this does not consist in tracking a mechanism's operation across time (not even in tracking multiple operations with different starting conditions) — although other explanations based on this mathematical model may do the latter.

While my discussion has focused on bifurcation analysis, I take it that there are other types of explanation used in systems biology that do not consist in tracking a mechanism's operation. Apart from topological explanations in terms of a network's structure (Huneman 2010) as mentioned in the introduction, there are also design explanations, which do not consist in a particular structure mechanistically explaining some functioning, but go in the opposite direction by the function explaining the class of structures that can realize the function (Braillard 2010; 
Brigandt et al. 2017). Systems biology generally explains the features of biological systems in terms of considerations about sensitivity or stability, capabilities and impossibilities, hierarchical control and the differential contributions made by different system components, leaving these diverse ways in which explanatory understanding is provided for future philosophical investigation.

\section{References}

Bechtel, William. 2010. "The Downs and Ups of Mechanistic Research: Circadian Rhythm Research as an Exemplar." Erkenntnis 73:313-28.

_. 2011. "Mechanism and Biological Explanation." Philosophy of Science 78:533-57.

—. 2012. "Understanding Endogenously Active Mechanisms: A Scientific and

Philosophical Challenge." European Journal for Philosophy of Science 2:233-48.

Bechtel, William, and Adele Abrahamsen. 2005. "Explanation: A Mechanist Alternative."

Studies in History and Philosophy of Biological and Biomedical Sciences 36:421-41.

—. 2010. "Dynamic Mechanistic Explanation: Computational Modeling of Circadian

Rhythms as an Exemplar for Cognitive Science." Studies in History and Philosophy of Science 41:321-33.

Braillard, Pierre-Alain. 2010. "Systems Biology and the Mechanistic Framework." History and Philosophy of the Life Sciences 32:43-62.

Brigandt, Ingo. 2013. "Systems Biology and the Integration of Mechanistic Explanation and Mathematical Explanation." Studies in History and Philosophy of Biological and Biomedical Sciences 44:477-92. 
_2015. "Evolutionary Developmental Biology and the Limits of Philosophical Accounts of Mechanistic Explanation." In Explanation in Biology: An Enquiry into the Diversity of Explanatory Patterns in the Life Sciences, ed. Pierre-Alain Braillard and Christophe Malaterre, 135-73. Dordrecht: Springer.

Brigandt, Ingo, Sara Green, and Maureen A. O’Malley. 2017. "Systems Biology and Mechanistic Explanation." In The Routledge Handbook of Mechanisms and Mechanical Philosophy, ed. Stuart Glennan and Phyllis Illari, 362-74. New York: Routledge.

Chen, Katherine C., Attila Csikasz-Nagy, Bela Gyorffy, John Val, Bela Novak, and John J. Tyson. 2000. "Kinetic Analysis of a Molecular Model of the Budding Yeast Cell Cycle." Molecular Biology of the Cell 11:369-91.

Craver, Carl F., and Lindley Darden. 2013. In Search of Mechanisms: Discoveries across the Life Sciences. Chicago: University of Chicago Press.

Eissing, Thomas, Holger Conzelmann, Ernst D. Gilles, Frank Allgöwer, Eric Bullinger, and Peter Scheurich. 2004. "Bistability Analyses of a Caspase Activation Model for ReceptorInduced Apoptosis." Journal of Biological Chemistry 279:36892-97.

Ferrell, James E., Jr. 2012. "Bistability, Bifurcations, and Waddington’s Epigenetic Landscape." Current Biology 22:R458-66.

Green, Sara, Maria Şerban, Raphael Scholl, Nicholaos Jones, Ingo Brigandt, and William Bechtel. 2017. "Network Analyses in Systems Biology: New Strategies for Dealing with Biological Complexity." Synthese. doi:10.1007/s11229-016-1307-6

Huneman, Philippe. 2010. "Topological Explanations and Robustness in Biological Sciences." Synthese 177:213-45.

— 2017. "Outlines of a Theory of Structural Explanations." Philosophical Studies. doi:10.1007/s11098-017-0887-4 
Issad, Tarik, and Christophe Malaterre. 2015. "Are Dynamic Mechanistic Explanations Still Mechanistic?" In Explanation in Biology: An Enquiry into the Diversity of Explanatory Patterns in the Life Sciences, ed. Pierre-Alain Braillard and Christophe Malaterre, 265-92. Dordrecht: Springer.

Legewie, Stefan, Nils Blüthgen, and Hanspeter Herzel. 2006. "Mathematical Modeling Identifies Inhibitors of Apoptosis as Mediators of Positive Feedback and Bistability." PLoS Computational Biology 2:e120.

Levy, Arnon, and William Bechtel. 2013. "Abstraction and the Organization of Mechanisms." Philosophy of Science 80:241-61.

— 2016. "Towards Mechanism 2.0: Expanding the Scope of Mechanistic Explanation." Paper presented at PSA 2016 (25th Biennial Meeting of the Philosophy of Science Association, Atlanta, November 3-6, 2016). http://philsci-archive.pitt.edu/id/eprint/12567 Novak, Bela, Attila Csikasz-Nagy, Bela Gyorffy, Kim Nasmyth, and John J. Tyson. 1998. "Model Scenarios for Evolution of the Eukaryotic Cell Cycle." Philosophical Transactions of the Royal Society B: Biological Sciences 353:2063-76.

Sterelny, Kim. 1996. "Explanatory Pluralism in Evolutionary Biology." Biology and Philosophy 11:193-214. 